\title{
Lifesaving Sonography Protocols: A Pilot Course Involving Undergraduate Medical Students
}

Jakub Wisniewski, ${ }^{1}$ Hanna Garnier.'

\begin{abstract}
Background: Ultrasonography protocols are easy to learn, frequently used in emergency medicine, and could be useful for inexperienced doctors. In this field, only a few protocols are needed to give an initial diagnosis and to start fast and proper treatment. Until now, only Focused Assessment with Sonography for Trauma (FAST) protocol training studies have been reported in the medical literature. Our point-of-care course, comprised of extended FAST, lung scan and ocular scan trainings. The students' curriculum usually does not include such ultrasonography courses, thus, we wanted to check its utility for the undergraduate medical students. Methods: Training lasted six days and consisted of two parts: 22 hours of theoretical classes and 18 hours of practical activities, all trained and evaluated by six experienced medical doctors. Eighty-five elected students completed pre- and post-study questionnaires about emergency ultrasonography and passed the practical final exam. Results: Eightyfive participants of the course were present in the pre- and final test. Final test scores of theoretical and practical exams were significantly higher after the training ( $58 \%$ vs. $87 \% ; n=85 ;$ p 0.01 ). Answers for the questions related to FAST and EFAST (extended FAST) were correct irrespective of completion of the course. A question regarding the sonographic evaluation of body fluid incontinence was found to be the most difficult for students. After the course, $96.5 \%$ of participants were able to complete an EFAST scan at an adequate level of performance in under two minutes. Conclusion: Results show that medical students significantly extended their knowledge about point-of-care emergency medicine ultrasonography and acquired practical skills during the course. Emergency medicine ultrasonography courses could be included into medical students' curricula.
\end{abstract}

Keywords: Ultrasonography; Education, Medical, Undergraduate; Education, Medical; Students, Medical (Source: MeSH-NLM).

\section{Introduction}

About the Author: Jakub Wisniewski is currently a final year medical student in a six year program at the Medical University of Gdansk, Gdansk, Poland. $\mathrm{He}$ is the President of Pe diatric Surgery and Urology Students' Scientific Association at the Medical University of Gdansk.

Submission: Jan 10, 2015 Acceptance: Mar 29, 2015

Process: Peer-reviewed and develop their clinical knowledge..$^{8-11}$ Ultrasonography use in EM seems to be one of the most important aspects. Some academics do not realize how important it can be in giving the prompt diagnosis, especially for the young doctors, who are still lacking experience. Ultrasonography allows fast clinical assessment of dehydration or can yield a prompt pneumothorax diagnosis. ${ }^{12,13}$ Until now, only FAST training studies have been reported in the medical literature. ${ }^{14}$ Our point-of-care course, included extended FAST, lung scan and ocular scan training.

Ultrasound examination and its correct interpretation require significant training and a lot of experience. ${ }^{2}$ However, in emergency medicine (EM), only a few protocols are needed to give an initial diagnosis and to start proper treatment. The most common protocol in the EM is Focus Assessment with Sonography for Trauma (FAST), which is only one part of the full ultrasound (US) examination. It gives a fast and accurate evaluation of the hemorrhage in the peritoneal cavity. ${ }^{3}$ Though adding ultrasonography to the students' curriculum seems to be time-consuming, it is proven that only two days ( 16 hours) of the course are needed to complete a correct FAST. ${ }^{4}$ Additionally, ultrasound examination of the eye (which allows for the rapid assessment of the cerebral edema) is easy enough to be taught and used by non-expert operators on the International Space Station (ISS).5,6

To date, there is an increasing number of ultrasound teaching pilot studies. These kinds of activities, used as a supplement to gross anatomy courses, are beneficial even during the first years of study. Many studies show that teaching ultrasonography to 3 rd, 4th, and 5 th year students can effectively support
We assumed that teaching easy protocols with brief training periods may be successfully included in the students' curriculum. The aim of our study was to check the effectiveness of the six-days course in EM for the medical students of the Medical University of Gdansk, Poland.

\section{Methods}

We conducted a six-days facultative course teaching about extended FAST (EFAST), Bedside Lung Ultrasound in Emergency (BLUE), Focus Assessed Transthoracic Echocardiography (FATE), monitoring of central venous access, ocular ultrasound in trauma and sonographic estimation of body fluid status assessment (Table 1). The training was held at the Medical University of Gdansk, Poland from October until November 2014.

Ninety-seven medical students of the University were voluntarily enrolled and they were requested to complete the pre-question-

${ }^{1}$ Final Year Medical Students, Medical University of Gdansk, Poland. 
Figure 1. Flow Diagram Showing Characteristics of the Study.

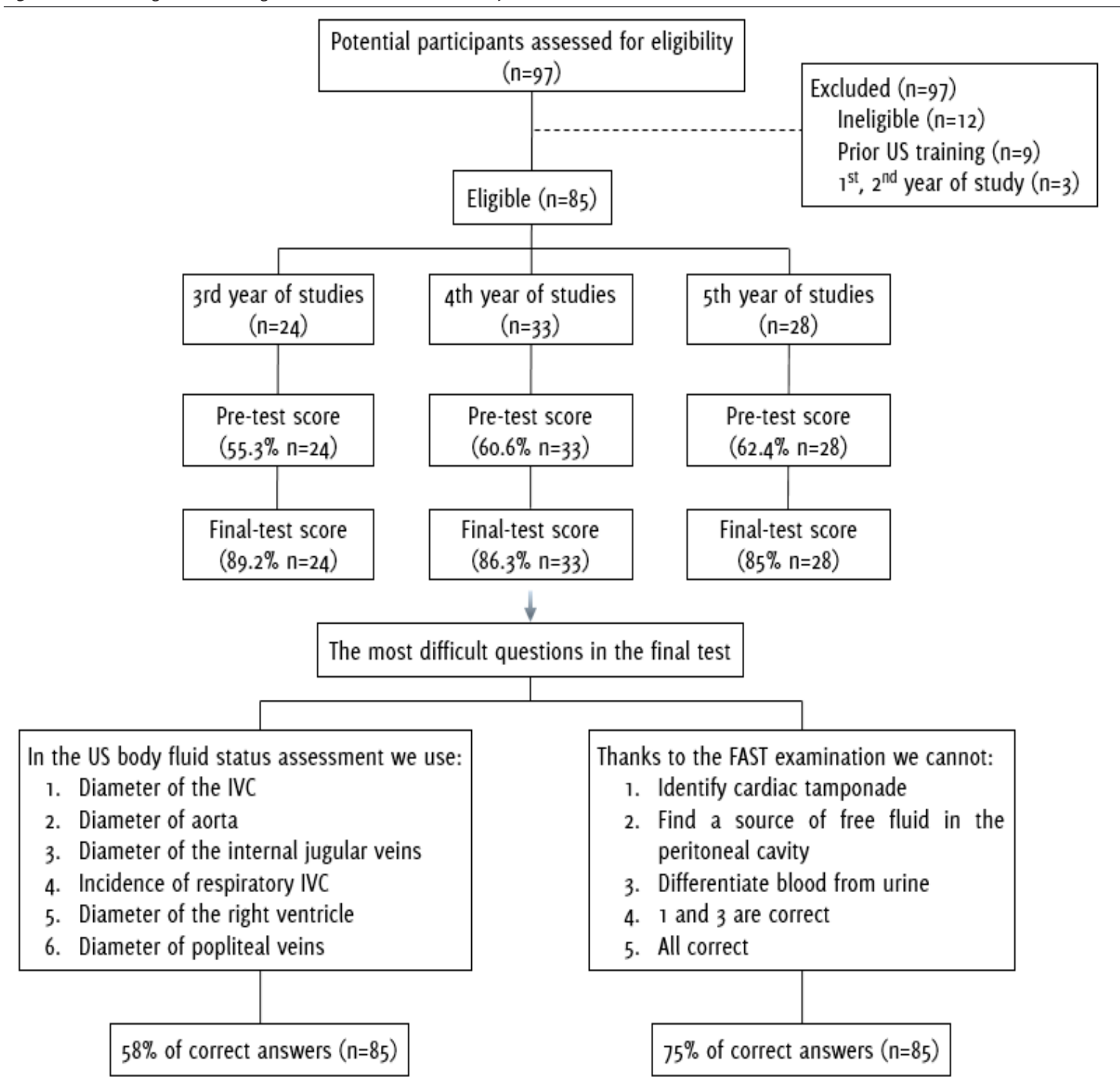

Legend: US - Ultrasound; IVC - inferior vena cava; FAST - Focused Assessment with Sonography for Trauma.

naire asking about their personal data and their previous experience with ultrasonography. Eighty-five from the 3 rd, 4 th, and 5 th years of study with no prior US course (inclusion criteria) were divided into four equal groups for the theoretical classes (Figure 1). Twelve students in the 1st and 2nd years of study or those with the prior US training were excluded. They were not informed about the course program in advance. Subsequently, each group was divided into four teams for practical training. We prepared a comprehensive, 6-days course for the medical students, focused on the use of an ultrasound in EM. The course consisted of two parts, 22 hours of the theoretical classes and 18 hours of the practical activities, all trained and evaluated by six experienced medical doctors. Before the course, students completed a multiple choice test of 25 questions about the practical use of ultrasound in EM. Eleven questions from the test involved knowledge about EFAST/FAST, five about ocular ultrasound in trauma, and five about BLUE. The same test was held after the course.

We analyzed all of the results from both of the tests (pre- and post-course) using a paired samples t-test and a $99 \%$ confiden- ce interval $(\mathrm{Cl}=99 \%)$. Statistical analyses were run using the STATISTICA 10 software (StatSoft Inc, Tulsa, OK).

After theoretical classes, students were working at cart-based ultrasonography machines (GE Logiq 7 and Philips: Sparq, ClearView, $\mathrm{CX}_{50}$, iU22). All of them were taught how to perform a proper EFAST, BLUE, FATE, and Ocular protocols. This part was evaluated by the practical test (EFAST scan completed in less than two minutes).

\section{Results}

Eighty-five participants in the course ( 23 from the 3 rd, 35 from the 4 th and 27 from the 5th year) participated in the pre- and final test.

The pretest mean scores of the students from the 3 rd, 4 th, and 5 th years were $56 \%(n=24), 61 \%(n=33)$, and $62 \%(n=28)$, respectively. The mean scores of the final test of the students from the $3 r d, 4$ th, and 5 th years were $89 \%(n=24), 86 \%(n=33)$, and $85 \%(n=28)$, respectively. There was no statistically significant differences between the scores of the students from the different years (pretest $p=0.32$; final test $p=0.47$ ). The total 
Figure 2. Comparison of the Pre And Final Test Results

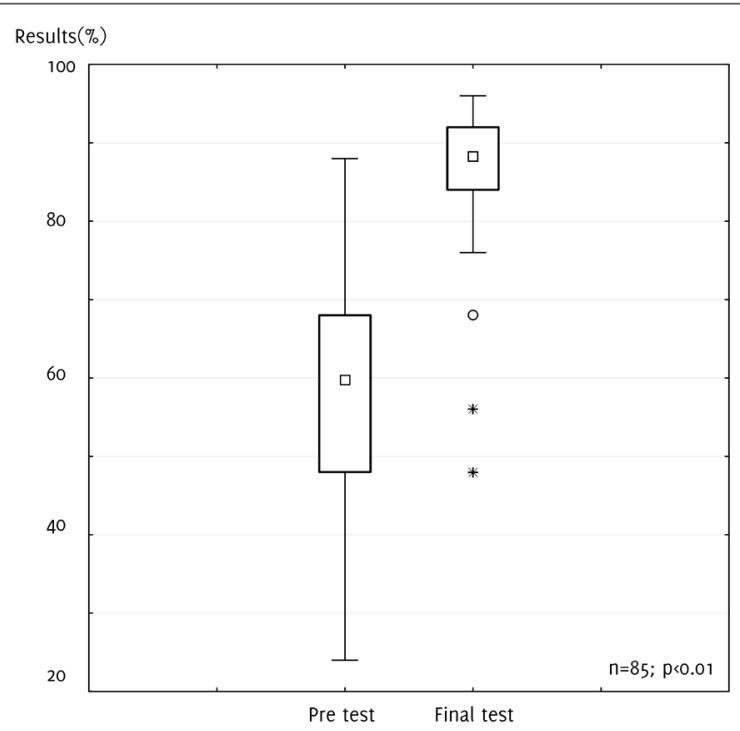

Legend: Data are presented as median (central square), 25-75\% (top and bottom of boxes). Top bar represents 1,5 of the highest interquartile range (IQR). Bottom bar represents 1,5 of the lowest IQR. The dot represents the middle outlier; stars represent two extreme outliers.

Table 1. Course Schedule

\begin{tabular}{|c|c|}
\hline Day & Topic \\
\hline $\begin{array}{l}\text { First } \\
\text { (Saturday) }\end{array}$ & $\begin{array}{l}\text { 1. From FAST* to ABCD ultrasound - introduction. } \\
\text { 2. US** in emergency medicine - for and against. } \\
\text { 3. I have an ultrasound equipment, what's next? Knobology. } \\
\text { 4. FAST } \\
\text { 5. E-FAST*** } \\
\text { 6. US and a central venous access. } \\
\text { 7. Polytrauma - ultrasongraphic diagnostic } \\
\text { 8. Patient in shock - ultrasonographic diagnostic } \\
\text { 9. Practical activities. }\end{array}$ \\
\hline $\begin{array}{l}\text { Second } \\
\text { (Monday) }\end{array}$ & $\begin{array}{l}\text { 1. Hydratation rating - how to use ultrasound. } \\
\text { 2. Practical activities. }\end{array}$ \\
\hline $\begin{array}{l}\text { Third } \\
\text { (Tuesday) }\end{array}$ & $\begin{array}{l}\text { 1. Other practical aspects of the US use in emergency medicine. } \\
\text { 2. Practical activities. }\end{array}$ \\
\hline $\begin{array}{l}\text { Fourth } \\
\text { (Wednesday) }\end{array}$ & $\begin{array}{l}\text { 1. BLUE' protocol. } \\
\text { 2. Practical activities. }\end{array}$ \\
\hline $\begin{array}{l}\text { Fifth } \\
\text { (Thursday) }\end{array}$ & $\begin{array}{l}\text { 1. } \text { FATE }^{\ddagger} \\
\text { 2. Practical activities. }\end{array}$ \\
\hline $\begin{array}{l}\text { Sixth } \\
\text { (Friday) }\end{array}$ & $\begin{array}{l}\text { 1. Final test. } \\
\text { 2. Case presentation. } \\
\text { 3. Discussion. } \\
\text { 4. Literature overview. } \\
\text { 5. Practical exam. }\end{array}$ \\
\hline
\end{tabular}

Legend: *Focused Assessment with Sonography for Trauma; **Ultrasonography; *** extended-FAST; †Bedside Lung US in Emergency; \#Focus Assessed Transthoracic Echocardiography.

mean final score $(86 \% ; n=85)$ was significantly higher $(p<0.01$; at statistical significance of level 0.01 ) than the mean result before the course ( $59.7 \% ; \mathrm{n}=85$ ) (Figure 2).

We found that most of the answers to the questions associated with FAST and EFAST were answered correctly irrespective of completion of the course. A question regarding the sonographic evaluation of body fluid incontinence turned out to be the most difficult. The second most difficult questioin was about the usa- ge of the EFAST examination. After the course, 96,5\% (82/85) of participants completed an EFAST scan at an adequate level of performance in under two minutes.

\section{Discussion}

Our six-days, intensive, point-of-care ultrasound course in EM turned out to be effective. Results showed that medical students significantly extended their knowledge about point-of-care EM US and acquired practical skills during the course. We can conclude that integrating emergency ultrasonography classes into medical school curriculum should be taken into consideration.

The pre-test results showed that students had elementary knowledge about ultrasonography before the course (acquired during radiology or emergency medicine classes). It suggests that the point-of-care ultrasound courses could be recommended as an additional training tool which helps to order and supplement the students' knowledge.

Ultrasonography, being an inexpensive and easy-to-use tool, can also be a "modern stethoscope" during daily ward rounds. It can have a particular importance, especially in the places where elderly staff have a skeptical opinion about the use of ultrasound in daily practice. More than $6 \%$ of the patients delivered to the emergency rooms are diagnosed with pneumotho$\mathrm{rax}^{15}$ and this diagnosis can be confirmed and localized with comparable efficacy to Computed Tomography in less than four minutes using ultrasound. ${ }^{16,17}$ This contributes to modernization and improvement in the hospital departments.

Regardless of numerous reports concerning the usefulness of ultrasonography in different educational stages, from studying anatomy support up to elderly medical students' education, it is still has not been entered into the curriculum. ${ }^{18}$ We need more studies (especially prospective) describing the effects of integrated ultrasonographic courses on the clinical practice. Medicine as a rapidly-developing science should pay attention, especially to the constant actualization of the students' curriculum. Thanks to this, young doctors will be able to skillfully use their medical knowledge in their future work.

The point-of-care ultrasound course has been added to the facultative curriculum at our University. Although, further research with longer follow-up should be done, in order to enhance the results and conclusions. According to the Kirkpatrick educational levels, we have evaluated our intervention at the level 2 (change in knowledge). To recommend change in the medical curriculum (which is already overcrowded), it would be necessary to reach at least level 3 evidence (change in behaviour or ability). A longer follow-up could help this stydy reach that level. ${ }^{19}$

Special guidelines are needed to unify both, the theoretical and practical training. There are no such instructions thus far. This is a limitation of our study; although we believe that Ultrasound Associations, such as European Federation of Societies for Ultrasound and Biology (EFSUMB; http://www.efsumb.org/) will publish adequate guidelines. That would help to create repeatable courses and would allow for the comparison of results from different universities in the world. Thus, further research should be done in order to determine whether our conclusions are correct across the globe.. 


\section{References}

1. Kosiak W. [Sonography as modern stethoscope in everyday practice of family doctors]. Fam Med Prim Care Rev. 2010;12(2):389-93. Polish.

2. Neri L, Storti E, Lichtenstein D. Toward an ultrasound curriculum for critical care medicine. Crit Care Med. 2007 May;35(5 Suppl):S290-304.

3. American Institute of Ultrasound in Medicine. AlUM practice guideline for the performance of the focused assessment with sonography for trauma (FAST) examination. J Ultrasound Med. 2014 Nov;33(11):2047-56.

4. Mohammad A, Hefny AF, Abu-Zidan FM. Focused Assessment Sonography for Trauma (FAST) training: a systematic review. World J Surg. 2014 May;38(5):1009-18.

5. Chiao L, Sharipov S, Sargsyan AE, Melton S, Hamilton DR, McFarlin K, et al. Ocular examination for trauma; clinical ultrasound aboard the International Space Station. J Trauma. 2005 May;58(5):885-9.

6. Martin DS, Caine TL, Matz T, Lee SM, Stenger MB, Sargsyan AE, et al. Virtual guidance as a tool to obtain diagnostic ultrasound for spaceflight and remote environments. Aviat Space Environ Med. 2012 Oct;83(10):995-1000.

7. Brown B, Adhikari S, Marx J, Lander L, Todd GL. Introduction of ultrasound into gross anatomy curriculum: perceptions of medical students. J Emerg Med. 2012 Dec;43(6):1098-102.

8. Swamy M, Searle RF. Anatomy teaching with portable ultrasound to medical students. BMC Med Educ. 2012 Oct 22;12:99.

9. Mircea PA, Badea R, Fodor D, Buzoianu AD. Using ultrasonography as a teaching support tool in undergraduate medical education - time to reach a decision. Med Ultrason. 2012 Sep;14(3):211-6.

10. Celebi N, Zwirner K, Lischner U, Bauder M, Ditthard K, Schürger S, et al. Student tutors are able to teach basic sonographic anatomy effectively - a prospective randomized controlled trial. Ultraschall Med. 2012 Apr:33(2):1415 .

11. Griksaitis MJ, Sawdon MA, Finn GM. Ultrasound and cadaveric prosections as methods for teaching cardiac anatomy: a comparative study. Anat Sci Educ. 2012 Jan-Feb;5(1):20-6.

12. Kosiak W, Swieton D, Piskunowicz M. Sonographic inferior vena cava/ aorta diameter index, a new approach to the body fluid status assessment in children and young adults in emergency ultrasound-preliminary study. Am J Emerg Med. 2008 Mar;26(3):320-5.

13. Lichtenstein D. Lung ultrasound in the critically ill. Curr Opin Crit Care. 2014 Jun;20(3):315-22.

14. Cogalniceanu P, Sheena Y, Kashef E, Purkayastha S, Darzi A, Paraskeva P. Is basic emergency ultrasound training feasible as part of standard undergraduate medical education? J Surg Educ. 2010 May-Jun;67(3):152-6.

15. Lichtenstein $D$, Axler 0 . Intensive use of general ultrasound in the intensive care unit. prospective study of 150 consecutive patients. Intensive Care Med. 1993 June; 19(6):353-5.

16. Kollef MH. Risk factors for the misdiagnosis of pneumothorax in the intensive care unit. Crit Care Med. 1991 Jul;19(7):906-10.

17. Ianniello S, Di Giacomo V, Sessa B, Miele V. First-line sonographic diagnosis of pneumothorax in major trauma: accuracy of e-FAST and comparison with multidetector computed tomography. Radiol Med. 2014 Sep;119(9):674-80.

18. Badea R. Medical education of students and residents - a new paradigm? Med Ultrason. 2012 Sep;14(3):175-6.

19. Abdulghani HM, Shaik SA, Khamis N, Al-Drees AA, Irshad M, Khalil MS, et al. Research methodology workshops evaluation using the Kirkpatrick's model: translating theory into practice. Med Teach. 2014 Apr;36(Suppl 1):S24-9.

\section{Acknowledgments}

We would like to thank Wojciech Kosiak MD, PhD and Tomasz Batko MD, PhD from the Departments of Paediatrics, Haematology, Oncology, and Endocrinology in the Medical University of Cdansk for organization of the course. We also thank Blazej Littwin MD, Andrzej Pomiecko and Marcin Gajewski for their invaluable support.

\section{Conflict of Interest Statement $\mathrm{C}$ Funding}

The Authors have no funding, financial relationships or conflicts of interest to disclose.

Author Contributions

Conception and design the work/idea, Collect data/obtaining results, Analysis and interpretation of data, Critical revision of the manuscript, Approval of the final version: JW, HG. Write the manuscript: JW. Statistical advice, Administrative or technical advice: HG.

Cite as:

Wisniewski J, Garnier H. Lifesaving Sonography Protocols: A Pilot Course Involving Undergraduate Medical Students. Int J Med Students. 2014 Nov-2015 Mar;3(1):15-8. 